\title{
EP-77
}

\section{Early experience of pure minimally invasive donor hepatectomy by junior surgeon}

\author{
Dai Hoon HAN${ }^{* 1}$, Jae Geun $\mathrm{LEE}^{2}$, Dong Jin $\mathrm{JOO}^{2}$, Gi Hong $\mathrm{CHOl}^{1}$, Jin Sub $\mathrm{CHOI}^{1}$ \\ 'Division of Hepatobiliary and Pancreatic Surgery, Department of Surgery, Yonsei University College of Medicine, Seoul, Korea \\ ${ }^{2}$ Division of Transplantation Surgery, Department of Surgery, Yonsei University College of Medicine, Seoul, Korea
}

Introduction: Although the pure minimally invasive donor hepatectomy (PMIDH) has been increasingly performed, it is still reported by experienced surgeons. Here we reported the early experience of PMIDM by junior surgeon.

Methods: From April 2019 to November 2020, PMIDHs were performed for 19 patients by single novice surgeon (H.D.H) at Yonsei University College of Medicine. Clinical characteristics, perioperative outcomes of donors and clinical features of recipients were analyzed.

Results: Eighteen of 19 donors had pure laparoscopic liver resection. Right, left hemihepatectomies and left lateral sectionectomies were performed for 15, 1 and 3 donors, respectively. Mean total operation time was $460.63 \pm 70.89$ minutes and median operative bleeding was $200 \mathrm{~mL}$ (50-1,600 mL). Median hospital stay was 8 (7-11) days. In terms of laparoscopic donor right hemihepatectomy, mean total operation time was $464.21 \pm 43.52$ minutes. Median amount of bleeding during operation was $225 \mathrm{~mL}(50-1,600 \mathrm{~mL})$. Mean estimated total liver volume was $1,228.26 \pm 217.01$ (935.80-1,584.90) and mean real graft weight was $764.86 \pm 153.46(529.00-$ 1,077.00). The robotic donor right hemihepatectomy was performed for one donor. Total operation time was $663 \mathrm{minutes}$ with $550 \mathrm{~mL}$ of operative bleeding. There was no conversion to open surgery. Only the first donor had transfusion during operation. There was no severe perioperative complication more than grade III according to classification of Clavien-Dindo surgical complication.

Conclusions: Although the PMIDH may need high level of surgical skill, it appears to be a safe and feasible procedure by junior surgeon in properly selected donors. 